\title{
Manejo preoperatorio de pacientes con Diabetes Mellitus*
}

\author{
Dr. CLAUDIO NAZAR J. ${ }^{1}$, Int. CHRISTIAN HERRERA F. ${ }^{2}$, Dr. ALEJANDRO GONZÁLEZ A. ${ }^{1}$
}

\author{
1 División de Anestesiología. \\ 2 Interno de Medicina. \\ Escuela de Medicina, Facultad de Medicina, Pontificia Universidad Católica de Chile. \\ Santiago, Chile.
}

\begin{abstract}
\section{Preoperative management of patients with Diabetes Mellitus}

Diabetic patients have a higher probability of being operated than the general population. Perioperative hyperglicemia is associated with the development of postoperative infections and cardiovascular complications. An adequate glycemic control reduces the risk for these complications. Therefore, the evaluation of preoperative hyperglicemia and target organ damage is of utmost importance among diabetic patients who will be subjected to surgical procedures. According to the results obtained, glycemic control can be optimized with dietary and pharmacological interventions.
\end{abstract}

Key words: Diabetes mellitus, surgery, complications.

\section{Resumen}

La Diabetes Mellitus es una condición crónica de hiperglicemia que afecta al 9,4\% de la población chilena. Estudios han encontrado que los pacientes con Diabetes Mellitus tienen mayor probabilidad de requerir cirugía en comparación a la población general. La hiperglicemia que presentan los pacientes se ha relacionado al desarrollo de complicaciones infecciosas y cardiovasculares en el período postoperatorio. Se ha demostrado que el adecuado control glicémico preoperatorio contribuye a disminuir el riesgo de desarrollar dichas complicaciones. Es por eso que se hace fundamental la evaluación preoperatoria para poder conocer los valores de glicemia que presenta el paciente y realizar los exámenes necesarios para determinar las consecuencias sistémicas que ha desarrollado la Diabetes Mellitus. De esta forma podremos realizar modificaciones en los tratamientos que utilizan los pacientes con el fin de optimizar su condición previo a la cirugía.

Palabras clave: Diabetes Mellitus 1 y 2, hiperglicemia, hipoglicemiantes orales, insulina, manejo preoperatorio.

*Recibido el 13 de noviembre de 2012 y aceptado para publicación el 3 de enero de 2013.

Los autores no refieren conflictos de interés

Correspondencia: Dr. Claudio Nazar J.

Marcoleta 367, 3er piso, Código postal: 6510260, Santiago, Chile.

cenazar@med.puc.cl 


\section{Introducción}

La Diabetes Mellitus (DM) es una condición crónica de hiperglicemia que afecta al $9,4 \%$ de la población chilena según la Encuesta Nacional de Salud del $2009^{1}$. Se ha visto un aumento sostenido en la prevalencia de DM en la población mundial. Los pacientes con esta patología tienen riesgo de desarrollar importantes complicaciones posteriores a un procedimiento quirúrgico tales como infecciones o enfermedad coronaria asintomática, lo que hace imperativo un adecuado control glicémico perioperatorio ${ }^{2,3}$.

Se estima que la prevalencia de DM es de $20 \%$ en pacientes quirúrgicos y $25 \%$ en pacientes hospitalizados ${ }^{2,4}$. Además se conoce que los pacientes diabéticos tienen un $50 \%$ de probabilidad de requerir cirugía de cualquier tipo alguna vez en la vida ${ }^{2,3}$.

Es importante evaluar el consumo de medicamentos en el período preoperatorio, ya que su uso por si mismo conlleva mayor riesgo de desarrollar complicaciones en el perioperatorio, de las cuales un $5 \%$ es atribuible a la suspensión del medicamento sin supervisión médica ${ }^{5}$, debido a la tendencia de los pacientes de suspender todos los fármacos previo a un procedimiento quirúrgico.

La literatura reconoce el efecto del acto quirúrgico en la condición metabólica del paciente, destacando el desarrollo de hiperglicemia y sus complicaciones asociadas en la evolución postoperatoria.

\section{Definición y diagnóstico de Diabetes Mellitus}

La DM es una condición metabólica crónica de hiperglicemia que resulta de la alteración en la secreción y/o acción de la insulina ${ }^{3}$. El estado de hiperglicemia crónica se asocia a largo plazo con disfunción e insuficiencia de diversos sistemas, especialmente el ocular, renal, tejido nervioso y cardiovascular ${ }^{4}$.

La alteración en el metabolismo se produce por anormalidades en el procesamiento de los hidratos de carbono, grasas y proteínas, dado por la acción deficiente de la insulina sobre los tejidos blanco. En un mismo paciente suele presentarse una alteración en la secreción de insulina y defectos en la acción de ésta.

Los síntomas característicos de hiperglicemia crónica son poliuria, pérdida de peso y polifagia. En tanto, condiciones agudas que pueden colocar en peligro la vida de un paciente diabético no contralado son la cetoacidosis diabética y el síndrome hiperosmolar no cetósico.

Existen diferentes tipos de DM, pero actualmente, más que importar catalogar al paciente en una de ellas, importa entender la patogénesis para un adecuado tratamiento en el período perioperatorio.

Los criterios diagnósticos para diabetes se resumen en la Tabla $1^{6}$, los cuales también son válidos en el contexto perioperatorio. Sin embargo, en un paciente sometido a cirugía los valores de hiperglicemia habitualmente son subestimados. Actualmente, han cobrado importancia los estados conocidos como prediabéticos (Tabla 2$)^{6}$, debido a que estos poseen un mayor riesgo cardiovascular y entre un $12 \%$ a $25 \%$ de probabilidad de desarrollar diabetes si dicha condición se mantiene ${ }^{6,7}$.

\section{Importancia del control glicémico preoperatorio}

Es necesaria una adecuada evaluación del paciente con DM previa a la cirugía para realizar los ajustes requeridos en caso de descontrol de la glicemia, ya sea hipoglicemia o hiperglicemia. Antes del año 2001, el principal riesgo de estos pacientes era el desarrollo de hipoglicemia inadvertida, debido al ayuno indicado, muchas veces 8-12 h antes de la cirugía y a la mantención de los fármacos hipoglicemiantes. Esto llevó a los cirujanos a permitir cirugías en contexto de hiperglicemias leves. Se ha demostrado que la hiperglicemia es un factor de riesgo para el desarrollo de infecciones postoperatorias y de complicaciones cardiovasculares, alterando la

Tabla 1. Criterios diagnósticos de Diabetes Mellitus ${ }^{6}$

Debe estar presente al menos uno de los siguientes:

1. Hemoglobina Glicosilada $\geq 6,5 \% * * *$

2. Glicemia de ayuno $\geq 126 \mathrm{mg} / \mathrm{dl}$ Requiere $8 \mathrm{~h}$ de ayuno***

3. Glicemia $\geq 200 \mathrm{mg} / \mathrm{dl} 2 \mathrm{~h}$ post carga de $75 \mathrm{~g}$ de glu$\cos a^{* * *}$

4. Paciente con síntomas clásicos de hiperglicemia, con glicemia aislada $\geq 200 \mathrm{mg} / \mathrm{dl}$

***En ausencia de hiperglicemia inequívoca, criterios 1-3 deben ser confirmados con repetición de la prueba.

Tabla 2. Estados con riesgo aumentado de desarrollar Diabetes Mellitus (Prediabetes) ${ }^{6,7}$

1. Glicemia de ayuno: $100 \mathrm{mg} / \mathrm{dl}-125 \mathrm{mg} / \mathrm{dl}$ (glicemia de ayuno alterada)

2. Test de tolerancia a glucosa: $140 \mathrm{mg} / \mathrm{dl}-199 \mathrm{mg} / \mathrm{dl} \mathrm{a}$ las $2 \mathrm{~h}$ posteriores a ingesta de $75 \mathrm{~g}$ de glucosa (intolerancia a la glucosa)

3. Hemoglobina glicosilada: $5,7 \%-6,4 \%$ 
respuesta inmune y produciendo un estado proinflamatorio en el período perioperatorio ${ }^{2-4,8-10}$.

Es reconocido que la cirugía produce respuesta a estrés en el paciente, desencadenando un desbalance de hormonas y citoquinas, favoreciendo un aumento de la glicemia, glucagón, catecolaminas, cortisol y resistencia a la insulina, lo que resulta en el desarrollo de hiperglicemia intra y/o postoperatoria.

De esta forma, los pacientes con hiperglicemia, ya sea por descontrol de su DM o por respuesta al estrés quirúrgico, presentan un mayor riesgo de morbilidad y mortalidad asociado al desarrollo de infecciones (de herida operatoria o pulmonares entre otras), accidentes cerebrovasculares, insuficiencia renal e isquemia miocárdica ${ }^{3,8}$. Esto es más evidente en los pacientes diabéticos que se someten a cirugía de by-pass coronario, en los cuales la mortalidad temprana post quirúrgica aumenta en $50 \%{ }^{8}$.

\section{Diabetes Mellitus y evaluación preoperatoria}

Sheehy y $\mathrm{col}^{7}$, han divido los estados hiperglicémicos de pacientes quirúrgicos en tres categorías. La primera de ellas agrupa a los pacientes con diagnóstico conocido de DM, Intolerancia a la Glucosa y Glicemia de Ayuno Alterada, en los cuales su estado es un factor de riesgo para el desarrollo de hiperglicemia. El grupo siguiente lo conforman los pacientes con DM sin diagnóstico conocido, quienes generalmente presentan hiperglicemia en el intraoperatorio, la que se mantiene hasta después del alta. Existe dificultad para diagnosticar a estos pacientes durante su hospitalización en la sala de cirugía ya que valores de hiperglicemia en este contexto pueden tener múltiples causas, siendo subvalorados en la práctica quirúrgica habitual. El estudio de una eventual DM debe ser realizado ambulatoriamente, previo a la cirugía programada. El último grupo incluye a los pacientes que presentan hiperglicemia inducida por estrés quirúrgico, situación que se resuelve espontáneamente una vez concluido el desbalance hormonal y el estado inflamatorio que se produce a causa de la cirugía. En este grupo, dicha condición no es predecible ni anticipable en la evaluación preoperatoria. No se debe pensar que toda hiperglicemia en un paciente sometido a cirugía sin antecedentes de DM sea secundaria al estrés quirúrgico ya que se corre el riesgo de subdiagnosticar dicha enfermedad metabólica.

Para optimizar el manejo perioperatorio de los pacientes con DM, Alexanian y co $^{10}$ proponen los siguientes objetivos para la evaluación preoperatoria:

1. Encontrar estados hiperglicémicos de acuerdo a lo explicado anteriormente y otras anormalidades metabólicas.
2. Controlar la glicemia con insulina en infusión endovenosa si es necesario.

3. Reconocer los pacientes quirúrgicos que desarrollen hipoglicemia.

4. Conocer la condición del paciente que será sometido a la intervención quirúrgica.

Respecto al objetivo glicémico durante el perioperatorio, se diferencia entre pacientes hospitalizados en condición crítica y aquellos que se encuentran estables. La importancia de esta división se basa en que la mayoría de los estudios de control glicémico se ha realizado en pacientes críticos, en los que se ha comparado un control estricto de la glicemia versus uno más holgado, encontrándose en el primer grupo un aumento significativo de la mortalidad y del desarrollo de hipoglicemia severa ${ }^{2,10-13}$. Si bien los valores de glicemia para cada grupo difieren entre los distintos trabajos, la Asociación Americana de Diabetes (ADA) recomienda lograr glicemias menores a $180 \mathrm{mg}$ en pacientes críticos y menores a $140 \mathrm{mg}$ en pacientes estables ${ }^{14}$.

La evaluación preoperatoria debe ser considerada un momento oportuno para la realización de tamizaje de DM según recomendaciones realizadas por la $\mathrm{ADA}^{6,7}$, quienes sugieren estudiar a todos los pacientes mayores de 45 años y aquellos menores de esta edad con índice de masa corporal (IMC) mayor o igual a $25 \mathrm{y}$ factores de riesgo adicionales que se mencionan en la Tabla 3.

\section{Manejo preoperatorio de medicamentos hipoglicemiantes}

Para un adecuado control metabólico prequirúrgico de los pacientes es necesario diferenciar entre los

Tabla 3. Pacientes en que se recomienda realización de pruebas de screening para Diabetes Mellitus ${ }^{6}$

1. Desde los 45 años en todo paciente

2. Pacientes con IMC mayor o igual a 25 con uno o más factores de riesgo:

- Sedentarismo

- Antecedente familiar primer grado con Diabetes

- Etnia de alto riesgo

- Mujeres con hijo nacido con macrosomía

- Hipertensión

- HDL menor a $35 \mathrm{mg} / \mathrm{dl}$ o triglicéridos mayor a $250 \mathrm{mgdl}$

- Mujeres con síndrome de ovario poliquístico

- Glucosa de ayuno alterada

- Intolerancia a la glucosa

- Condiciones de resistencia a la insulina

- Historia de enfermedad cardiovascular. 
dos principales tipos de DM y el tipo de tratamiento que este paciente mantiene en forma crónica. Como recomendación general, los pacientes diabéticos deben tener su cirugía a primera hora del día para disminuir las interrupciones de su manejo basal cuando se inicia el ayuno ${ }^{3}$.

Respecto a los exámenes de laboratorio, éstos deben ser solicitados para conocer el control glicémico con el que se presenta el paciente a la cirugía y buscando alteraciones cardiovasculares y renales. Se recomienda el uso de hemoglobina glicosilada para evaluar el control glicémico en los tres meses previos a la cirugía. Para conocer la función cardiovascular y renal se debe solicitar un electrocardiograma en reposo en busca de indicadores de isquemia miocárdica previa o actual y creatinina sérica para evaluar daño renal crónico, ya que ambos son factores de riesgo para el desarrollo de eventos cardiovasculares e insuficiencia renal en el postoperatorio ${ }^{2,3,7,8}$. Los resultados de dichos exámenes deben ser interpretados a la luz de sintomatología previa del paciente que sugiera enfermedad cardiovascular o renal.

\section{Diabetes Tipo 2 tratado sólo con dieta}

Estos pacientes no requieren un manejo perioperatorio particular ${ }^{3}$. Se recomienda controlar glicemia en el preoperatorio y precozmente al finalizar la cirugía y, si es necesario, corregir los niveles de glucosa sanguínea usando insulina subcutánea según el esquema de la Tabla $4^{2}$.

\section{Diabetes Tipo 2 tratado con hipoglicemiantes orales}

Los hipoglicemiantes orales se dividen en tres grupos ${ }^{4}$ :

1) Fármacos que aumentan la secreción de insulina (sulfonilureas y no sulfonilureas).

2) Drogas que aumenta la sensibilidad a la insulina (biguanidas y tiazolidinedionas).

3) Nuevas drogas tales como agonistas del péptido símil a glucagón tipo 1 (GLP-1) e inhibidores de la dipetidil peptidasa IV (DPP-4).

\section{Grupo 1: Drogas que aumentan secreción de insulina}

Sulfonilureas (glipizide y tolbutamida). Actúan aumentado la secreción de insulina desde las células $\beta$ del páncreas. Tienen vida media larga, alcanzando hasta $72 \mathrm{~h}$, por lo que aumentan el riesgo de desarrollar hipoglicemia durante el ayuno. Si bien en estudios de laboratorio estos medicamentos han demostrado disminuir el riesgo de isquemia miocárdica, esto no ha sido comprobado por ensayos clínicos, en los cuales su uso ha mostrado empeoramiento en la morbilidad y mortalidad cardiovascular. Dado lo anterior, se recomienda suspender las sulfonilureas la mañana de la cirugía, o bien, una vez que el paciente ha iniciado el ayuno dado el riesgo de presentar hipoglicemia sintomática preoperatoria ${ }^{2-4,8,15-17}$.

No Sulfonilureas (repaglinida, nateglinide y mitiglinide). También aumentan la secreción de insulina, pero a través de un mecanismo diferente al de las sulfonilureas. Tienen menor vida media, alcanzando su máxima acción desde los 30 a 60 min post administración, lo que permite su uso con cada comida. Recomendamos suspender estos medicamentos luego de administrar la dosis que acompaña la última ingesta de alimentos antes de iniciar el ayuno ${ }^{4}$ ("última comida, última dosis").

\section{Grupo 2: Drogas que aumentan la sensibilidad a la insulina}

Biguanidas. La metformina, único representante de este grupo, actúa en los hepatocitos disminuyendo la producción hepática de glucosa $\mathrm{y}$, a nivel periférico, aumentando el depósito de ésta. Uno de los efectos adversos es la acidosis láctica en pacientes con deterioro de la función renal, lo que se asocia a mortalidad desde $30 \%$ hasta $50 \%$. En vista de lo anterior, se recomienda suspender la metformina 1 a 2 días antes de una cirugía electiva, sobre todo si se sospecha que habrá inestabilidad hemodinámica o alteración de la perfusión renal durante el procedimiento, como por ejemplo, gran cirugía vascular $^{2-4,8,15-17}$.

Tiazolidinedionas (pioglitazona y rosiglitazona). Actúan a nivel de un receptor nuclear, modulando el metabolismo de carbohidratos y lípidos en tejido adiposo, muscular y hepático. Son de acción corta (vida media de 3 a $4 \mathrm{~h}$ ), pero para alcanzar su efecto máximo requieren de uso continuo por 6 a 12 semanas. Dentro de sus efectos adversos destaca la retención hídrica y la formación de edema periférico, el que se presenta en 3\% a 5\% de los pacientes. Además, puede producir anemia dilucional, edema pulmonar (1\% a $3 \%$ de los pacientes) e insuficiencia cardíaca congestiva. No existen estudios que comparen los efectos entre su continuación o término de uso en el período preoperatorio inmediato, pero dado que sus efectos adversos pueden entorpecer la evolución postoperatoria se recomienda suspender su uso varios días antes de la cirugía ${ }^{2-4,8}$. La evidencia médica disponible no especifica la cantidad de días necesarios de suspensión, por lo que se requieren más estudios para responder esta interrogante.

\section{Grupo 3: Nuevas Drogas}

Análogos de GLP-1 (exentide). El GLP-1 es un péptido endógeno que aumenta la secreción de insulina ante la ingesta de glucosa, inhibe la liberación de glucagón y retrasa el vaciamiento gástrico. 
Tabla 4. Esquema de suplementación de Insulina para corrección de hiperglicemia ${ }^{2}$

\begin{tabular}{|cccc|}
\hline $\begin{array}{c}\text { Glicemia } \\
(\mathbf{m g} / \mathbf{d l})\end{array}$ & $\begin{array}{c}\text { Sensibilidad a insulina (U) } \\
\text { (Pacientes adultos mayores, diabéticos } \\
\text { tipo 1 e insuficientes renales) }\end{array}$ & $\begin{array}{c}\text { Normal } \\
(\mathbf{U})\end{array}$ & $\begin{array}{c}\text { Resistencia a insulina (U) } \\
\text { (Pacientes obesos o usuarios } \\
\text { de corticoides) }\end{array}$ \\
\hline $141-180$ & 2 & 4 & 6 \\
$181-220$ & 4 & 6 & 8 \\
$221-260$ & 6 & 8 & 10 \\
$261-300$ & 8 & 10 & 12 \\
$301-350$ & 10 & 12 & 14 \\
$351-400$ & 12 & 14 & 16 \\
$>400$ & 14 & 16 & 18 \\
\hline
\end{tabular}

Además, se ha postulado un probable efecto de inducción en la proliferación de células $\beta$ del páncreas.

Inhibidores de DPP-IV (sitagliptina). La función de esta droga consiste en bloquear la degradación del GLP-1.

Estos dos últimos medicamentos no producen hipoglicemia en estados de euglicemia dado su mecanismo de acción, por lo que pueden ser administrados el mismo día de la cirugía ${ }^{2-4}$.

Si los pacientes poseen un buen control metabólico ambulatorio, la suspensión de los hipoglicemiantes orales durante el período perioperatorio no debiera desencadenar hiperglicemia, pero en caso de presentarla, se puede utilizar insulina subcutánea de acción corta según lo indicado en la Tabla $4^{2-4}$.

\section{Diabetes tipo 1 y 2 tratados con insulina}

El uso de insulina busca reproducir la secreción fisiológica de esta hormona. Se recomienda mantener el mismo esquema que utiliza el paciente mientras se prosigue con la ingesta oral y continuar con el uso de insulina durante el período perioperatorio, pero adaptando su forma de administración según el tiempo que tomará el acto quirúrgico y la complejidad de la intervención a la que será sometido el paciente:

- Si el procedimiento es breve y de baja complejidad puede continuar con la administración de insulina por vía subcutánea. En caso que el paciente utilice insulina NPH, lenta o glargina, la noche previa a la cirugía se debe administrar dos tercios de la dosis habitual nocturna y la mitad de la dosis matinal habitual en la mañana del procedimiento. Lo anterior es fundamental para los pacientes con DM Tipo 1 con el fin de prevenir el desarrollo de cetoacidosis ${ }^{2-4}$. Además, para evitar los efectos metabólicos del ayuno, se recomienda iniciar suero glucosado al 5\%, a una velocidad de 75 a $125 \mathrm{ml} / \mathrm{h}$, lo que aporta 3,75 a 6,25 gramos de glucosa/hora ${ }^{3,18}$.
- Para cirugías de mayor duración y alta complejidad se recomienda el uso de insulina por infusión endovenosa, ya que ha demostrado un mejor control glicémico y una rápida titulación en caso de ser necesario ${ }^{3,4,18,19}$. Se utiliza insulina regular y la cantidad a administrar se obtiene al dividir por 24 la dosis total diaria de insulina usada en forma ambulatoria, lo que aproximadamente corresponde a $0,02 \mathrm{U} / \mathrm{kg} / \mathrm{h}$. Se administra junto a una solución salina a concentración $0,1 \mathrm{U} / \mathrm{ml}$, lo que se logra con $50 \mathrm{U}$ de insulina regular en 500 $\mathrm{ml}$ de esta solución. Esta concentración puede aumentarse en caso de que se necesite menos volumen debido a estados de hipervolemia del paciente.

Con el fin de evitar la aparición de hipoglicemia y para entregar sustrato calórico, se aporta glucosa en forma de suero glucosado al $5 \%$ a $100-150$ $\mathrm{ml} / \mathrm{h}$. Junto a esto se administra potasio $(20 \mathrm{mEq} / \mathrm{L}$ de $\mathrm{KCl}$ ) con cada litro de volumen para prevenir hipokalemia debido a la acción de la insulina. La infusión debe iniciarse al menos $2 \mathrm{~h}$ previas a la cirugía y no antes, ya que con dicho tiempo basta para alcanzar un buen control glicémico. Durante la cirugía, se controla glicemia cada $1 \mathrm{~h}$ con hemoglucotest mientras se mantenga la infusión endovenosa de insulina. Por otro lado, el potasio se controla cada $6 \mathrm{~h}$.

\section{Conclusiones}

La DM es una condición de hiperglicemia prevalente en la población y quienes la padecen tienen mayor probabilidad de requerir una cirugía en algún momento de su vida. El equipo quirúrgico debe conocer las implicancias de la hiperglicemia en el desarrollo de complicaciones en el período perioperatorio y como prevenirlas logrando un adecuado 
control glicémico. Se han estudiado dos tipos de control, uno estricto y otro más holgado, encontrando mayor mortalidad y desarrollo de hipoglicemia en los pacientes que fueron sometidos a control estricto. Los niveles de glicemia considerados óptimos son distintos según la gravedad del paciente, así en aquellos que se encuentran en condición crítica se buscan glicemias menores a $180 \mathrm{mg} / \mathrm{dl}$ y $140 \mathrm{mg} /$ dl en los pacientes estables. Para un adecuado enfrentamiento de estos pacientes resulta útil dividirlos según el tratamiento ambulatorio usado. Los hipoglicemiantes orales deben ser suspendidos según su vida media y el riesgo de producir hipoglicemia perioperatoria. Los pacientes que utilizan insulina deben continuarla, pero se ajusta su dosis y vía de administración según el tipo de cirugía al cual será sometido el paciente.

\section{Referencias}

1. Ministerio de Salud Gobierno de Chile, Facultad de Medicina Pontificia Universidad Católica de Chile, Observatorio Social Universidad Alberto Hurtado. Encuesta nacional de salud 2009-2010 Tomo II, V. Resultados, 2.4 Diabetes Mellitus:138-51.

2. Meneghini L. Perioperative management of diabetes: translating evidence into practice. Cleve Clin J Med. 2009;76:53-9.

3. Khan N, Ghali W, Cagliero E. Perioperative management of diabetes mellitus. UpToDate Inc. Disponible en www.uptodate.com (consultado el 22 de abril de 2012).

4. Tuttnauer A, Levin P. Diabetes mellitus and anesthesia. Anesthesiol Clin. 2006;24:579-97.

5. Kennedy J, van Rij A, Spears G, Pettigrew R, Tucker I. Polypharmacy in a general surgical unit and consequences of drug withdrawal. Br J Clin Pharmacol. 2000;49:353-62.

6. American Diabetes Association. Diagnosis and Classification of Diabetes Mellitus. Diabetes Care 2012;35:6471.

7. Sheehy A, Gabbay R. An overview of preoperative glu- cose evaluation, management, and perioperative impact. J Diabetes Sci Technol. 2009;3:1261-9.

8. Robertshaw H, Hall G. Diabetes mellitus: anaesthetic management. Anaesthesia 2006;61:1187-90.

9. Maerz L, Akhtar S. Perioperative glycemic management in 2011: paradigm shifts. Curr Opin Crit Care 2011;17:370-5.

10. Alexanian S, McDonnell M, Akhtar S. Creating a perioperative glycemic control program. Anesthesiol Res Pract. 2011;2011:465974.

11. Bhamidipati C, LaPar D, Stukenborg G, Morrison C, Kern J, Kron I, et al. Superiority of moderate control of hyperglycemia to tight control in patients undergoing coronary artery bypass grafting. J Thorac Cardiovasc Surg. 2011;141:543-51.

12. Kansagara D, Fu R, Freeman M, Wolf F, Helfand M. Intensive insulin therapy in hospitalized patients: a systematic review. Ann Intern Med. 2011;154:268-82.

13. Desai S, Henry L, Holmes S, Hunt S, Martin C, Hebsur $\mathrm{S}$, et al. Strict versus liberal target range for perioperative glucose in patients undergoing coronary artery bypass grafting: a prospective randomized controlled trial. J Thorac Cardiovasc Surg. 2012;143:318-25.

14. American Diabetes Association. Executive Summary: Standards of Medical Care in Diabetes-2012. Diabetes Care 2012;35:4-10.

15. Juvany R, Mercadal G, Jodar R. Manejo perioperatorio de la medicación crónica no relacionada con la cirugía. An Med Interna 2004;21:291-300.

16. Castanheira L, Fresco P, Macedo A. Guidelines for the management of chronic medication in the perioperative period: systematic review and formal consensus. J Clin Pharm Ther. 2011;36:446-67.

17. Saber W. Perioperative medication management: a casebased review of general principles. Clevel Clin J Med. 2006;73:82-7.

18. Glister B, Vigersky R. Perioperative management of type 1 diabetes mellitus. Endocrinol Metab Clin North Am. 2003;32:411-36.

19. Schiff R, Welsh G. Perioperative evaluation and management of the patient with endocrine dysfunction. Med Clin North Am. 2003;87:175-92. 